\title{
Fluorescent CdSe/ZnS Nanocrystal-Peptide Conjugates for Long-term, Nontoxic Imaging and Nuclear Targeting in Living Cells
}

\author{
Fanqing Chen ${ }^{1, \S, *}$, Daniele Gerion ${ }^{2, \S}$ \\ ${ }^{1}$ Life Sciences Division, Lawrence Berkeley National Laboratory, Berkeley, CA 94720; \\ ${ }^{2}$ Physics and Advanced Technology, Lawrence Livermore National Laboratory, Livermore, CA \\ 94551 \\ ${ }^{\S}$ F.C and D.G. contributed equally. (f_chen@lbl.gov, gerion1@1lnl.gov) \\ *To whom correspondence should be addressed. \\ Life Sciences Division \\ Lawrence Berkeley National Laboratory \\ MS 74R0157, 1 Cyclotron Rd. \\ Berkeley, CA 94720
}

KEYWORDS: Quantum dots, Live cell, imaging, nuclear localization, nanocrystal 


\section{Abstract}

One of the biggest challenges in cell biology is the imaging of living cells. For this purpose, the most commonly used visualization tool is fluorescent markers. However, conventional labels, such as organic fluorescent dyes or green fluorescent proteins (GFP), lack the photostability to allow the tracking of cellular events that happen over minutes to days. In addition, they are either toxic to cells (dyes), or difficult to construct and manipulate (GFP). We report here the use of a new class of fluorescent labels, silanized CdSe/ZnS nanocrystal-peptide conjugates, for imaging the nuclei of living cells. CdSe/ZnS nanocrystals, or so called quantum dots (qdots), are extremely photostable, and have been used extensively in cellular imaging of fixed cells. However, most of the studies about living cells so far have been concerned only with particle entry into the cytoplasm or the localization of receptors on the cell membrane. Specific targeting of qdots to the nucleus of living cells has not been reported in previous studies, due to the lack of a targeting mechanism and proper particle size. Here we demonstrate for the first time the construction of a CdSe/ZnS nanocrystal-peptide conjugate that carries the SV40 large T antigen nuclear localization signal (NLS), and the transfection of the complex into living cells. By a novel adaptation of commonly used cell transfection techniques for qdots, we were able to introduce and retain the NLS-qdots conjugate in living cells for up to a week without detectable negative cellular effects. Moreover, we can visualize the movement of the $\mathrm{CdSe} / \mathrm{ZnS}$ nanocrystal-peptide conjugates from cytoplasm to the nucleus, and the accumulation of the complex in the cell nucleus, over a long observation time period. This report opens the door for using qdots to visualize long-term biological events that happen in the cell nucleus, and provides a new nontoxic, long-term imaging platform for nuclear trafficking mechanisms and cell nuclear processes. 
To understand the complexity and dynamics of cellular events in living organisms, it is desirable to image the nucleic acids, proteins, or metabolites inside living cells. In live cell imaging, the entry of the probe into the nucleus and its visualization constitute increasingly important areas of research ${ }^{1,2}$. The nucleus is a desirable target because the genomic DNA, which carries the genetic information of the cell, resides there. In addition, numerous nuclear proteins actively participate in critical cellular processes such as DNA replication, recombination, RNA transcription, DNA damage and repair, genomic alterations, and cell cycle control. The efficient transport of probes into the nuclei of living cells would greatly enhance the diagnosis of disease genotype, the tracking of oligonucleotide drugs, the understanding of biological processing in the nucleus, and the identification of potential nuclear drug candidates. However, in living cells, a double-membrane nuclear envelope separates the cytoplasm from the cell nucleus. This physical barrier is impermeable to most kinds of probes, except at specific locations, a few tens of nanometers wide, called the nuclear pores ${ }^{3}$.

Currently, for imaging living cells, fluorescent tagging with organic fluorophores ${ }^{4}$ or green fluorescent protein $^{5}$ (GFP) is still the most commonly used method. Unfortunately, organic dyes are usually toxic to the cells and therefore the use of organic fluorophores for live cell applications has obvious limitations. Moreover, organic dyes and GFP suffer from notorious shortcomings such as photobleaching, which preclude their use in many long-term imaging applications. These fluorophores also have limited sensitivity and resolution, both of which are critical factors for accurate tracking of individual biomolecules. Finally, recombinant GFP fusion proteins are cumbersome to construct, and long-term imaging (>3days) with GFP requires the time-consuming process of establishing stable-expressing clones. 
To solve the stability and sensitivity issue, other types of labels such as polymeric ${ }^{6}$, magnetic ${ }^{7}$ and metallic ${ }^{8-10}$ particles have been introduced into cells. However, fluorescence microscopy remains the simplest and most used detection tool, and it would therefore be desirable to develop a technology based on robust fluorescent probes. Inorganic semiconductor nanocrystals, or qdots, represent this alternative technology ${ }^{11}$. Qdots, such as CdSe/ZnS core/shell nanoparticles, are inorganic fluorophores with a size below $10 \mathrm{~nm}$. Compared to conventional dyes, they have a much higher photobleaching threshold and negligible photobleaching under biological imaging conditions. Qdots can be silanized ${ }^{12}$ and, in that form, have reduced phototoxicity and are highly resistant to chemical and metabolic degradation ${ }^{13}$. Finally, whereas the organic fluorophores require customized chemistry for conjugation of biomolecules to each fluorophore, a universal approach can be used for the conjugation of biomolecules to all silanized qdots, because the silica shell coatings for different qdots are identical.

Unlike technologies based on gold nanoparticles or organic labels, the use of qdot labels is still in its infancy. Yet this technology is progressing at a fast pace ${ }^{14,15}$. Recently, a wide variety of biomolecules such as DNA ${ }^{16,17}$, proteins, antibodies ${ }^{18,19}$, short peptides ${ }^{20}$, and neurotransmitters ${ }^{21}$ have been attached to qdots. For instance, qdots have been used extensively as immunohistochemical labels in fixed cells. In vitro, qdots conjugated to immunoglobin $\mathrm{G}$ (IgG) have been used for the detection of membrane proteins such as the cancer marker Her2. The study of living cells presents an additional difficulty, viz., the introduction of the qdots inside the cells. Different methods have been reported. The crawling over a qdot-coated collagen surface allows the living cells to engulf the nanoparticles, which permits the study of their motility patterns ${ }^{13}$. Microinjection into Xenopus embryo has been used to follow cell dynamics during embryogenesis ${ }^{17}$. Finally, receptor-mediated endocytosis has also been used to transfect 
living cells. For instance, qdots bearing Epidermal Growth Factor (EGF) have been demonstrated to bind to erbB/Her receptors and are actively endocytosed into endosomes in living cells ${ }^{22}$.

Even though in vitro and in vivo imaging with qdots has been demonstrated, most of these studies have focused on the entry of dots into the cytoplasm or targeting of the membrane proteins ${ }^{15,17-19,21}$. Detection of nuclear proteins has been reported only in fixed cells by using anti-nuclear antigens ${ }^{19}$. Qdots has also been shown to accumulate in cell nucleus by passive diffusion after cell division ${ }^{17}$ (ref 17. Norris et al. (Qdots-2004, Banff, Canada, this is the reference suggested by the reviewer, I think we should include it). However, so far, no report has investigated the feasibility of active and targeted localization of qdots into the nuclei of living cells. The challenges for the use of qdots for targeted nuclear delivery are multiple ${ }^{2,9,23,}$ ${ }^{24}$. First, qdots must have a surface chemistry that allows their escape from endosomal/lysosomal pathways in living cells. Second, qdots must possess a nuclear localization signal (NLS) to be transported by the nuclear trafficking proteins and interact with the nuclear pore complex. Third, the diameter of the nuclear pore complex is $20-50 \mathrm{~nm}$ depending on the cell line ${ }^{3}$, and therefore the qdot conjugates have to be small enough $(<20 \mathrm{~nm})$ to cross the nuclear membrane. Finally, qdot conjugates must not interfere with normal physiology of the cells. In addition, the qdot conjugates must enter the cell via transfection or receptor-mediated endocytosis rather than through microinjection, so that a significant number of cells can be studied.

The most efficient nuclear targeting in biology is accomplished by viruses, which commonly utilize different peptides for crossing the nuclear membrane barrier ${ }^{2,25}$. For example, signal peptides from SV40 large T antigen and HIV Tat protein are effective in nuclear translocation of recombinant fusion proteins. Our strategy has thus been to make use of the nuclear localization 
signal from the SV-40 large $\mathrm{T}$ antigen. In this study, we create a compact $(\sim 10-15 \mathrm{~nm})$ complex with the SV40 nuclear localization signal peptide attached to a qdot, hereafter called "NLSqdot." Labeling with qdots does not interfere with the growth or differentiation of the cells. Using an extremely convenient transfection-based delivery technique developed for peptide-qdot conjugates, we have successfully introduced these conjugates into the human Hela cells and retained them for weeks. We have observed the accumulation of the NLS-qdots preferentially in the cell nucleus or in the perinuclear region. In contrast, qdots conjugated to a random peptide sequence showed almost homogeneous distribution within the cytoplasm, did not localizein any preferred region of the cell, and were always excluded from the nucleus. Also, with a simple fluorescent microscopy setup, we were able to continuously image and track movement of NLSqdots in live cells for more than $1 \mathrm{hr}$.

Silanized qdots (emission $\sim 550 \mathrm{~nm}$, fwhm $\sim 35 \mathrm{~nm}$ ) are prepared according to methods reported in the literature ${ }^{12}$. Their average size is about $8-10 \mathrm{~nm}$. The peptide sequences are bound to the silanized qdots through a streptavidin-biotin bridge, as indicated in Fig.1A and its caption. First, streptavidin-maleimide (Sigma) is covalently linked to the thiols of silanized dots. We use a ratio STV:qdot of 2:1 to $4: 1$ in a $10 \mathrm{mM}$ phosphate buffer, $\mathrm{pH} \sim 7$ with $10 \%$ of formamide, and overnight reaction. Excess of unbound STV is removed by several runs of centrifugation in a Centricon 100 device. The linking of STV to the qdots is probed by an assay where non-fluorescent biotinylated microbeads are incubated with STV-qdot solutions. Microbeads exposed to STV-qdots exhibit a fluorescent signal characteristic of the STV-qdot conjugates. Hence, microbeads exposed to red STV-qdot fluoresce in red and those exposed to green STV-qdots fluoresce in green. Control experiments, where STV is missing on the qdots or where it is blocked by an excess of biotin, show no fluorescence (Fig. 1B). The STV-qdot 
conjugates are then linked to biotinylated NLS sequences $\left[\mathrm{N}_{\text {terminal }}-\right.$ ( Pro Pro Lys Lys Lys Arg Lys Val ) ${ }_{2}-\mathrm{C}_{\text {terminal }}$ Biotin] or to a biotinylated random peptide sequence [biotin $\mathrm{N}_{\text {terminal }}$ Glu Pro

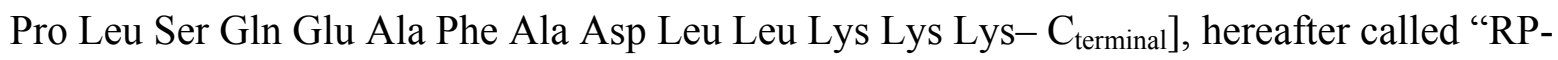
qdots". This conjugation step is performed in $10 \mathrm{mM}$ phosphate buffer, $150 \mathrm{mM} \mathrm{NaCl}, \mathrm{pH} 7.3$ at room temperature. For labeling experiments we used a ratio of peptide:qdots of 5:1 to 10:1 with 20 min of reaction time. The incorporation of biotinylated peptides onto the STV-qdot conjugates under these conditions is best probed by gel electrophoresis, where the ratios peptide:qdots spans from 1:1 to 50:1. Figure 1C illustrates the titration curve of the conjugation between the qdots and the NLS sequence. Negatively charged STV-qdot conjugates exhibit a reduced mobility when incubated with the biotinylated NLS peptide and the overall mobility decreases as the concentration of NLS increases (lane 1 to 8). At a ratio of 50 NLS per qdot, the NLS-qdot conjugates do not move from the well, yet such complexes do not show sign of bulk aggregation for weeks. Also, the post-addition of free biotin to the NLS-qdot conjugates that do not migrate restores a partial mobility to the qdots. Given the timeframe for such an experiment (30 min of electrophoresis), it is very unlikely that such pattern represents diffusion of the particles in the gel. Rather, we believe that free biotin is able to displace some biotinylated-NLS, reducing thus the number of positively charged peptides bound to qdots and increasing the NLSqdot mobility. This implies that, to a large extend, the NLS peptide does not bind nonspecifically to STV-qdots. An additional indication in that direction is the fact that if free biotin saturates the STV binding sites of STV-qdots, the addition of a 50-fold excess of NLS has a very slight effect on the qdot mobility (lane 10 and lane 12).

While the attachment of the peptide to qdots can be studied by test-tube experiments, their activity cannot. The only way to probe the biological activity of NLS-qdot conjugates is to 
check if the complexes are indeed able to target the cell nucleus. In this regard, the first step is to find an efficient way of introducing the peptide-qdot conjugates inside a large number of living cells. We have investigated two methods: electroporation and lipofectamine transfection. However, in this paper we will focus only on electroporation and comment on lipofactamine transfection in supplement information. We have investigated the electroporation of 2 types of qdots: NLS-qdots, qdots conjugated to SV40 NLS; and RP-qdots, qdots conjugated to the random peptide.

Our cell model system consists of human HeLa cells grown as monolayer cultures in a humidified, $5 \% \mathrm{CO}_{2}$ atmosphere with $\alpha$-minimal essential medium supplemented with $10 \%$ heatinactivated fetal calf serum. The cells were trypsinized, resuspended in PBS, counted using a Coulter Counter and diluted at $1 \times 10^{5}$ cells $/ \mathrm{ml}$. Qdots were mixed with the cells at a ratio of 10 pmol of qdots $/ 1 \times 10^{5}$ cells, for a final concentration of $10 \mathrm{nM}$ of qdots and $1 \mathrm{ml}$ total volume. Electroporation was carried out in a $4 \mathrm{~mm}$ electroporation cuvette (Bio-Rad, CA) using 300V, $250 \mu \mathrm{F}$, and a pulse time of 5-6 msec with a Gene-Pulser II (Bio-Rad, CA). The cells were then seeded on the slide chamber (NUNC). Attachment of the cells to the slide surface was achieved with either centrifugation in a Beckman benchtop centrifuge at a speed of 1000 rpm for 2 minutes or by natural sedimentation for at least 2 hours.

Figure 2 shows HeLa cells transfected with NLS-qdots viewed under a 100x oil immersion objective $^{26}$. For comparison, Fig. 3 shows cells transfected with qdots conjugated to a random peptide sequence. All images are taken $24 \mathrm{hrs}$ after transfection. The NLS-qdot conjugates are observed either in the cell nucleus (Fig. 2, panel A) or in the perinuclear region (Fig. 2, panel B). The percentage of cells with NLS-qdots localized in the nucleus is $\sim 15 \%$, while in $\sim 85 \%$ of the cases, NLS-qdots accumulate preferentially in the perinuclear region. In contrast, qdots 
conjugated to a random peptide localize randomly in the cells (Fig.3) and we do not see these conjugates inside the nucleus within the $24 \mathrm{hr}$ time frame. The qualitative and distinct localization of the NLS-qdots and RP-qdots seems to preclude a passive mechanism, such as free diffusion, for the qdots entry into the nucleus, as it was previously observed as a result of disruption of nuclear membrane during multiple cell division cycles ${ }^{17}$. Remarkably, when the NLS-qdot conjugates are in the nucleus, finer structures within the nucleus, such as the nucleoli, are revealed (Fig. 2A, far left panel, one nucleolus is indicated by the arrow).

An intriguing question remains as to why certain NLS-qdots can all enter the nucleus of some HeLa cells, while NLS-qdots from the same transfection get all stuck in the perinuclear region of some other cells. Since the same NLS-qdot conjugates are used, the partial aggregation of the dots or their incorporation into vesicles although possible is not the main reason. Rather, a rough estimate indicates that the NLS-qdots may have an overall size close to the nuclear pore sizes. In this case, intrinsic characteristics of the nuclear pores (size, shape, permeability, etc) may become a dominant factor for the qdot entry. Indeed, a possible explanation invokes the variation of plasticity of the nuclear membrane during the cell cycle. The rate of nuclear pore formation of HeLa cells has been shown to vary with the cell cycle ${ }^{27}$. In addition, the newly formed pores comprise a subpopulation that are more permeable than mature ones. Because asynchronous cells population was used in this study, we propose that HeLa cells with NLSqdots in their nucleus represent a subpopulation of cells at a particular stage of the cell cycle.

The visualization of the routes of NLS-qdot movement provide a mean to better understand the protein nuclear trafficking process mediated by nuclear localization signal, in this case, the SV40 NLS. While images of RP-qdots are mainly static in time, the fact that NLS-qdots actively seek to enter the nucleus can be visualized by tracking them. The general direction of 
movements of the NLS-qdots goes from the periphery of the cytoplasm to the perinuclear region (Fig. 4A). However, at the nuclear membrane, some NLS-qdots remain stuck, while some apparently enter the nucleus. This is better illustrated in Fig. 4B, where two types of complexes are indicated by arrows. A qualitative feature of fluorescence distinguishes them. Some fluorescent spots (red arrow in Fig. 4B) are large and bright, photobrighten with time, and remain in close contact with the nuclear membrane. They are likely vesicles containing a large collection of NLS-qdots. Other spots are smaller and blurred, and weaker in intensity, but the intensity is stable over time (yellow and white arrows). We associate these latter features to single NLS-qdots or to aggregates of a small number of NLS-qdots, since these are the features that are observed to enter the nucleus.

The NLS-qdots demonstrate extreme chemical and photostability in the cells. We measured the fluorescence from NLS-qdot conjugates in the cells as a function of time under continuous excitation. The electroporation (Fig. 5) did not decrease the NLS-qdot fluorescence, and in some cases, we even observed an increase in the signal. As mentioned previously, such photobrightening is observed for the brighter spots, which we tentatively attribute to vesicles or aggregates of qdots. In addition to short-term monitoring of individual cells, we also followed the transfected cells for a prolonged period, and the fluorescent signals from NLS-qdots were still present in the cells after a week. A picture of two cells just after cell division at day 5 is shown in Fig. 6A.

In their elemental form, cadmium, selenium, zinc, and sulphur have all been known to cause acute and chronic toxicities in living organisms. The cellular toxicity of surfactant-stabilized

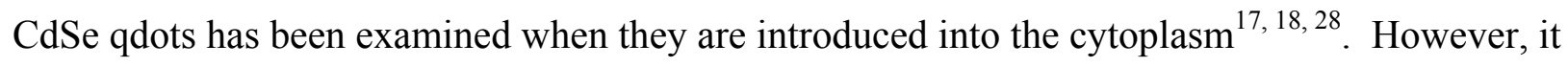
is the cell nucleus that contains the genetic material DNA and the transcriptional machinery of 
the cell, and these are more sensitive to permanent alterations and damages. No data yet exist on the toxicity of the NLS- and STV-qdots introduced into the nucleus. To demonstrate that the cells are not adversely affected by the NLS-qdots, we assayed for cytotoxicity by comparing colony-forming capability of the transfected cells vs. sham-transfected cells ${ }^{29}$. The survival of the cells carrying different doses of qdots was compared with cells transfected with PBS only. The dose of qdots applied here are $100,10,1$ pmole $/ 10^{6}$ cells. We found that the nuclear accumulation of NLS-qdots had minimal impact on cellular survival (Fig. 6B). This indicates that the silica coating of the qdots has successfully prevented the interaction of $\mathrm{Cd}, \mathrm{Se}, \mathrm{Zn}$, and $\mathrm{S}$ to the proteins and DNA in the nucleus. The introduction of streptavidin and the SV40 nuclear localization signal, at the concentrations used in this study, have no adverse effect on cell survival. Close to $100 \%$ of transfected cells survived in all the experiments, indicating that the cytotoxicity caused by the transfection procedure is negligible.

In summary, we have constructed a peptide-qdot conjugate that has the ability to actively translocate to the cell nucleus. The conjugate has the advantage of low cytotoxicity, higher sensitivity, desirable photostability, long-term biological stability, resistance to lysosomal degradation, and reasonable resistance to aggregation within the cell. The presence of NLS may facilitate the active entry of qdots through the nuclear pore complex. The size of the NLS-qdots conjugate seems a critical factor in trafficking efficiency. Because of the thick layers of coating added by the silanization process, current commercial qdots fall in the size range of well above $25 \mathrm{~nm}$ when streptavidin and additional peptides are added, and their bigger size precludes them from entering the nucleus. The smaller size of silanized NLS-qdot conjugates used here might explain the reason why the earlier STV-qdots from commercial source did not transverse the nuclear membrane, the nuclear pore complex playing the gatekeeper that prevented transport to 
the nucleus. Smaller qdots might have a better nuclear transport profile. The natural extension of the conjugates developed here will be the addition of antibodies specific to nuclear proteins to the NLS-qdot complex. This will be useful in imaging targeted nuclear proteins and important biological processes in the nucleus. In addition to the SV40 large T antigen nuclear localization signal, there are other NLS that may work more efficiently for qdots, such as the Tat NLS sequence from HIV. Further improvement of nuclear localization can be achieved. Nuclear localization signal from other nuclear proteins can also be tagged to qdot to facilitate the study of nuclear trafficking mechanisms employed by these proteins. The electrical charges on the surface of the nanoparticles could also play an important role in determining the transfection and localization properties. The charges on the surface of the silanized qdots can be altered ${ }^{30}$, and the proteins that carry the NLS-qdots to the nucleus may have different levels of affinity to qdots with a different surface electrostatic charge. We are also actively investigating whether the same techniques can be used to target other organelles in the cell system, such as ER, Golgi, membrane, proteasome, perixisome, or mitochondria, with qdots bearing the proper signal peptides.

\section{Acknowledgment}

We thank Dr. Joe W. Gray for comments on the manuscript, Drs. N. Zaitseva and G. Galli for their support, and Prof. A.P. Alivisatos for access to his laboratory. This work was supported by NIH Grant R21CA95393-01, by NASA grant NNA04CA75I, and by Department of Energy grant to F. Chen. This work was performed under the auspices of the U.S. Dept. of Energy, at the University of California/Lawrence Livermore National Laboratory under contract no. W-7405Eng-48, and at the University of California/Lawrence Berkeley National Laboratory under 
contract no. DE-AC03-76SF00098. 


\section{REFERENCES}

(1) Jans, D. A.; Chan, C. K.; Huebner, S. Medicinal Research Reviews 1998, 18, 189-223.

(2) Jans, D. A.; Xiao, C. Y.; Lam, M. H. C. Bioessays 2000, 22, 532-544.

(3) Fahrenkrog, B.; Aebi, U. Nature Reviews Molecular Cell Biology 2003, 4, 757-766.

(4) Swedlow, J. R.; Platani, M. Cell Structure and Function 2002, 27, 335-341.

(5) Ehrhardt, D. Current Opinion in Plant Biology 2003, 6, 622-628.

(6) Liu, J. Q.; Zhang, Q.; Remsen, E. E.; Wooley, K. L. Biomacromolecules 2001, 2, 362368.

(7) Hinds, K. A.; Hill, J. M.; Shapiro, E. M.; Laukkanen, M. O.; Silva, A. C.; Combs, C. A.; Varney, T. R.; Balaban, R. S.; Koretsky, A. P.; Dunbar, C. E. Blood 2003, 102, 867-872.

(8) Cognet, L.; Tardin, C.; Boyer, D.; Choquet, D.; Tamarat, P.; Lounis, B. Proceedings of the National Academy of Sciences of the United States of America 2003, 100, 1135011355.

(9) Tkachenko, A. G.; Xie, H.; Coleman, D.; Glomm, W.; Ryan, J.; Anderson, M. F.; Franzen, S.; Feldheim, D. L. Journal of the American Chemical Society 2003, 125, 47004701.

(10) Schultz, S.; Smith, D. R.; Mock, J. J.; Schultz, D. A. Proceedings of the National Academy of Sciences of the United States of America 2000, 97, 996-1001.

(11) Bruchez, M., Jr.; Moronne, M.; Gin, P.; Weiss, S.; Alivisatos, A. P. Science 1998, 281, 2013-2016.

(12) Gerion, D.; Pinaud, F.; Williams, S. C.; Parak, W. J.; Zanchet, D.; Weiss, S.; Alivisatos, A. P. Journal of Physical Chemistry B 2001, 105, 8861-8871.

(13) Pellegrino, T.; Parak, W. J.; Boudreau, R.; Le Gros, M. A.; Gerion, D.; Alivisatos, A. P.; Larabell, C. A. Differentiation 2003, 71, 542-548.

(14) Alivisatos, P. Nature Biotechnology 2004, 22, 47-52.

(15) Dahan, M.; Levi, S.; Luccardini, C.; Rostaing, P.; Riveau, B.; Triller, A. Science 2003, 302, 442-445.

(16) Gerion, D.; Chen, F.; Kannan, B.; Fu, A.; Parak, W. J.; Chen, D. J.; Majumdar, A.; Alivisatos, A. P. Anal Chem 2003, 75, 4766-4772.

(17) Dubertret, B.; Skourides, P.; Norris, D. J.; Noireaux, V.; Brivanlou, A. H.; Libchaber, A. Science 2002, 298, 1759-1762.

(18) Jaiswal, J. K.; Mattoussi, H.; Mauro, J. M.; Simon, S. M. Nat Biotechnol 2003, 21, 47-51.

(19) Wu, X.; Liu, H.; Liu, J.; Haley, K. N.; Treadway, J. A.; Larson, J. P.; Ge, N.; Peale, F.; Bruchez, M. P. Nat Biotechnol 2003, 21, 41-46.

(20) Akerman, M. E.; Chan, W. C.; Laakkonen, P.; Bhatia, S. N.; Ruoslahti, E. Proc Natl Acad Sci U S A 2002, 99, 12617-12621.

(21) Rosenthal, S. J.; Tomlinson, A.; Adkins, E. M.; Schroeter, S.; Adams, S.; Swafford, L.; McBride, J.; Wang, Y. Q.; DeFelice, L. J.; Blakely, R. D. Journal of the American Chemical Society 2002, 124, 4586-4594.

(22) Lidke, D. S.; Nagy, P.; Heintzmann, R.; Arndt-Jovin, D. J.; Post, J. N.; Grecco, H. E.; Jares-Erijman, E. A.; Jovin, T. M. Nat Biotechnol 2004, 22, 198-203.

(23) Suh, J.; Wirtz, D.; Hanes, J. Proceedings of the National Academy of Sciences of the United States of America 2003, 100, 3878-3882. 
(24) Goldfarb, D. S.; Gariepy, J.; Schoolnik, G.; Kornberg, R. D. Nature 1986, 322, 641-644.

(25) Whittaker, G. R. Advanced Drug Delivery Reviews 2003, 55, 733-747.

(26) The imaging of cells was performed by fluorescence imaging with an upright Olympus microscope BX51. The illumination source was a Hg lamp, and images were recorded with a Peltier-cooled CCD camera. Integration time varied from $200 \mathrm{msec}$ to $700 \mathrm{msec}$ per frame. For the tracking of the movement of qdots, the cells were illuminated continuously for periods of up to 1 hour, and images were taken every 15 seconds and subsequently processed.

(27) Feldherr, C. M.; Akin, D. Journal of Cell Biology 1990, 111, 1-8.

(28) Derfus, A. M.; Chan, W. C. W.; Bhatia, S. N. Nano Letters 2004, 4, 11-18.

(29) Cells transfected with NLS-qdot complexes at different concentrations or with PBS only were plated at density of 300 cells $/ 60 \mathrm{~mm}$ dish and allowed to grow for 10 days. Colonies formed by individual cells were stained by Crystal Violet dye and counted by ColCount (Oxford Optronix, Oxford, UK), with the relative number of surviving colonies used as an index for cell vitality.

(30) Parak, W. J.; Gerion, D.; Zanchet, D.; Woerz, A. S.; Pellegrino, T.; Micheel, C.; Williams, S. C.; Seitz, M.; Bruehl, R. E.; Bryant, Z.; Bustamante, C.; Bertozzi, C. R.; Alivisatos, A. P. Chemistry of Materials 2002, 14, 2113-2119. 


\section{Figure Captions}

Figure 1. Preparation of silanized NLS-qdot conjugates with SV40 nuclear signal and RP-qdots conjugates with a random peptide sequence. A) Schematic of the dots used. From left to right: silanized qdots, streptavidin-qdot conjugates (STV-qdot) and qdot conjugated to a peptide through a streptavidin-biotin bridge. B) Probing the formation of STV-qdot intermediate. The binding of two colors of STV-qdots to biotinylated microbeads produces fluorescent beads. On the right, in the control experiments, the microbeads do not fluorescence because the qdots do not have STV or because STV is blocked by an excess of free biotin. Scale bar: $10 \mu \mathrm{m} . \mathbf{C}$ ) Incorporation of the biotinylated NLS sequence on the STV-qdots. The ratio of NLS:qdots is 0, $1,3,5,10,15,20,50,50,50,0,0$. The mobility is reduced as the NLS content increases. In lanes 9 and 10, free biotin is added to the reaction. In lane 9, biotin is added 15 minutes after the NLS, and is kept reacting for 15 additional minutes prior to running the gel. Notice how free biotin seems capable of displacing the NLS from the STV-qdots. In lane 10, free biotin was added prior to the NLS addition and saturated the STV binding site. The presence of a 50 -fold excess of NLS does not affect markedly the mobility of STV-qdots whose binding sites are blocked by biotin (lane 12). This suggests that at concentrations used for transfection (peptide:qdot=5:1), the non-specific binding of the peptide is negligible.

Figure 2. Localization of NLS-qdots in the nucleus (A) or in the perinuclear region $(\mathbf{B})$. In certain cases, such as the cell at the far left of panel A, NLS-qdots localized inside the nucleus reveal finer structures such as nucleoli within the nucleus, one of which is indicated with the arrow. Scale bars: $10 \mu \mathrm{m}$.

Figure 3. Qdots conjugated to a random peptide sequence, or RP-qdots, localize in random regions of the cells, including in the perinuclear region but never inside the nucleus. Scale bars: $10 \mu \mathrm{m}$.

Figure 4. Movement of NLS-qdots conjugates. A) False-color overlay of fluorescence from a cell taken at $4 \mathrm{~min}$ interval, about $2 \mathrm{hr}$ after transfection. In 4 minutes, the dots moved from the green to the red position. The features at the top of the image go downwards and the features on the bottom go upwards. Notice the movement of the fluorescent dots at the bottom of the image towards the nucleus. Integration time: $700 \mathrm{msec}$. B) An example of a large aggregate of NLSqdots is indicated with the red arrow. This feature is immobile during the entire period of illumination (15 min). In contrast, smaller, weaker, blurred yet distinguishable features move continuously during the measurement. The pathways of two of these spots are shown. The positions of the spots at the beginning of the measurement are indicated by the circled stars and the arrows. Positions are determined every $15 \mathrm{~s}$ for a total time of 15 minutes. Integration time: $500 \mathrm{msec} /$ frame. Scale bar: $5 \mu \mathrm{m}$.

Figure 5. Stability of the fluorescence signal as a function of time for the transfection by electroporation. Two types of behaviors are represented here. First, features similar to the one indicated by a red arrow in Fig. 4 b exhibit a marked photobrightening $(\boldsymbol{\Delta})$. Such features are likely vesicles or aggregates containing a large number of NLS-qdots. Second, features similar to the moving conjugates in Fig. $4 \mathrm{~b}$ exhibit a stable signal for up to $1 \mathrm{hr}(\boldsymbol{\square}$ and $\circ)$. 
Figure 6. Cell division and Cytotoxicity assays. A) The image shows the division of a cell, 5 days after transfection of the NLS-qdots. Notice the localization of the NLS-qdots in the nuclei of the two newly divided cells in the view field. B) Cell survival measured by colonigenic assay. Three hundred transfected cells were seeded per $100 \mathrm{~mm}$ tissue culture plate, and allowed to grow for 10 days to form colonies. The numbers of surviving cells are recorded counting the numbers of colonies formed. The concentrations of NLS-qdots used are: $100 \mathrm{pmole} / 10^{6}$ cells, 10 pmole $/ 10^{6}$ cells, 1 pmole $/ 10^{6}$ cells, and no NLS-qdot treatment for negative control; the final concentrations are 100,10,1 nM. The cells transfected with NLS-qdots showed a statistically insignificant difference from the cells transfected with just PBS. The transfection procedure itself had minimal impact on the survival of the cells. On average, more than $90 \%$ of the cells survived the transfection. 
A. Scheme for qdot conjugates

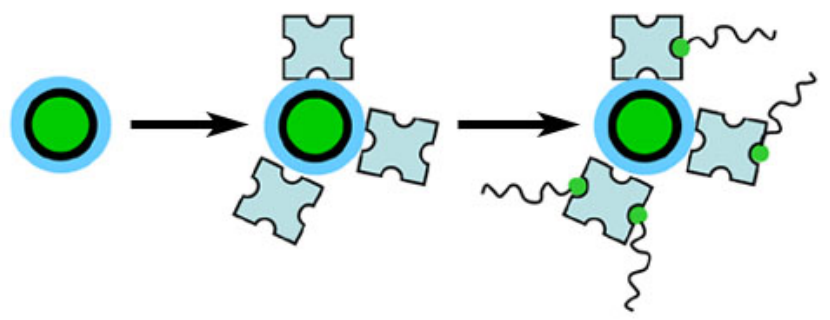

B. Formation of STV-qdots

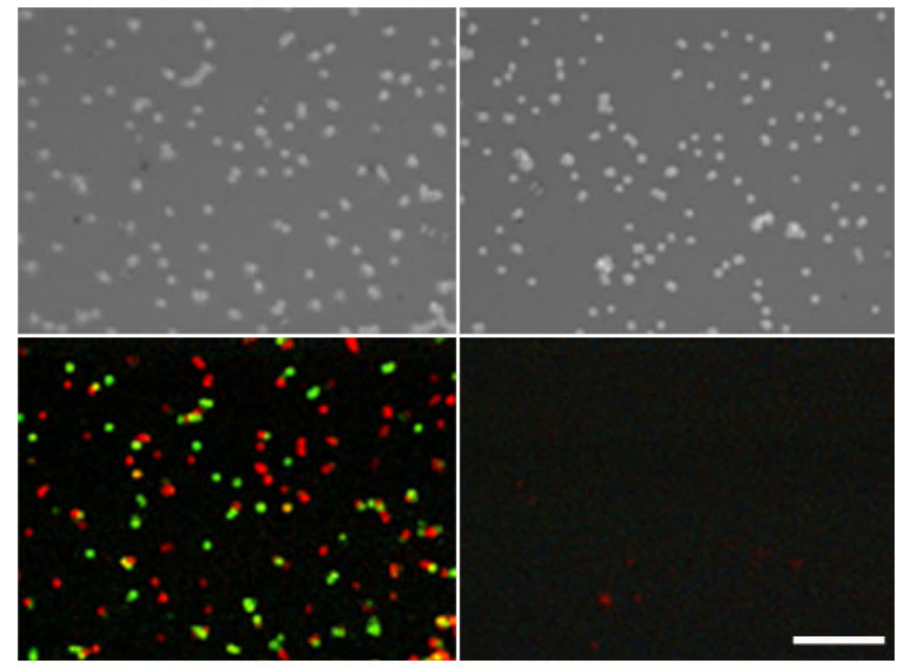

C. Formation of NLS-qdots

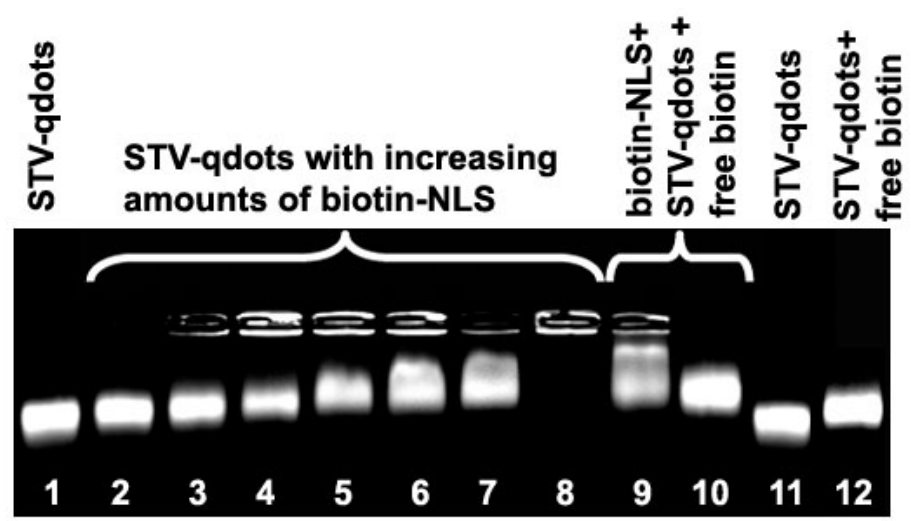

Figure 1 

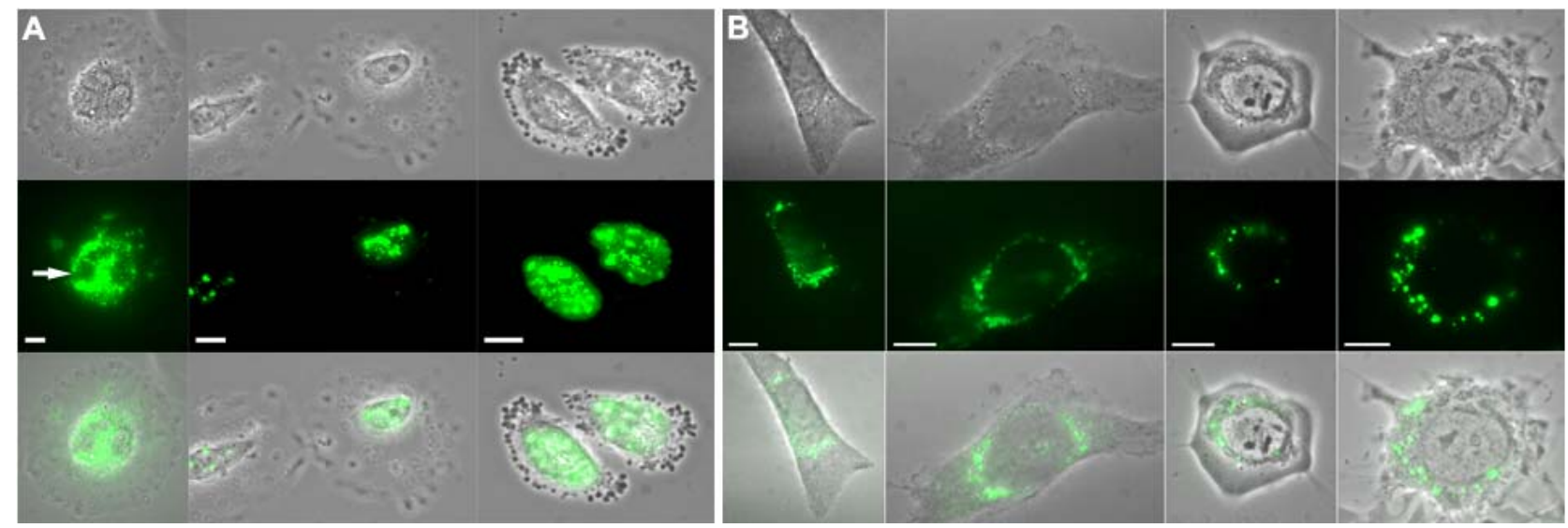

Figure 2

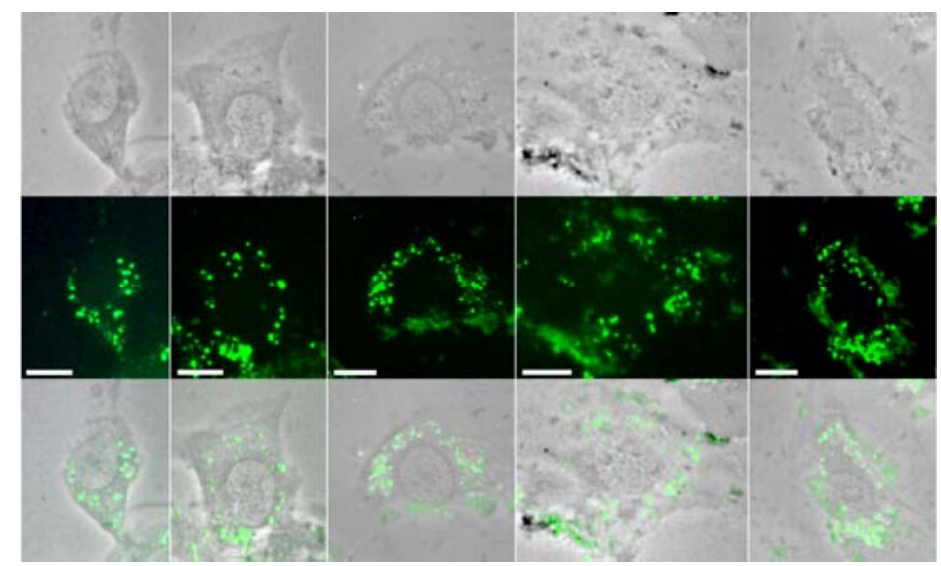

Figure 3 


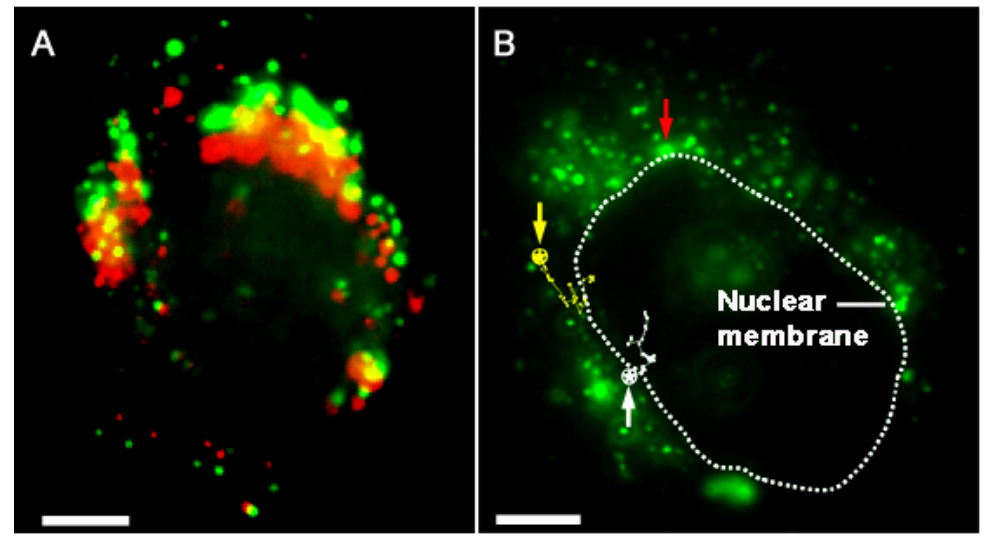

Figure 4

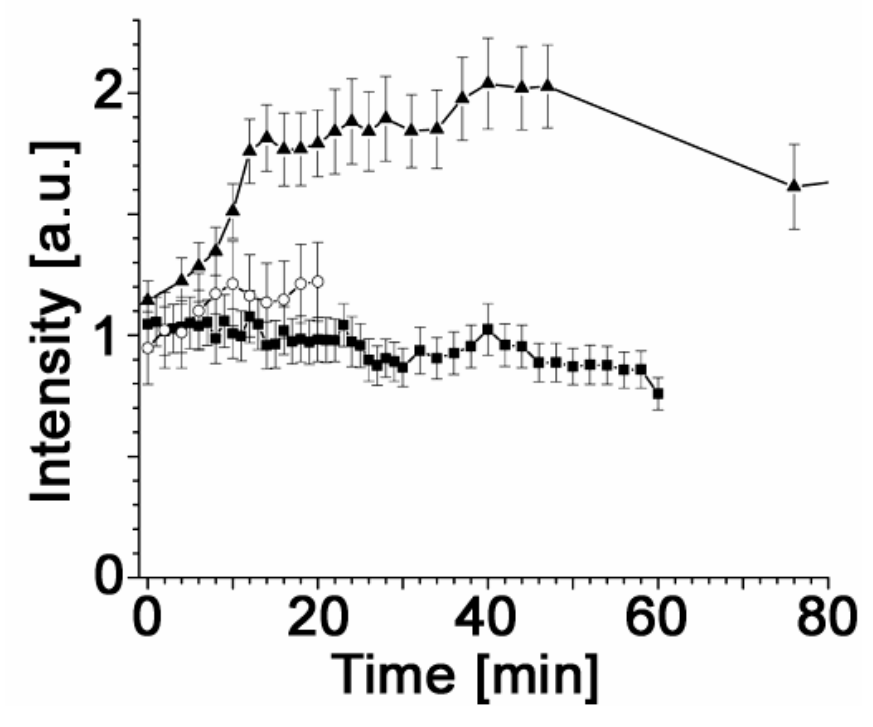

Figure 5 


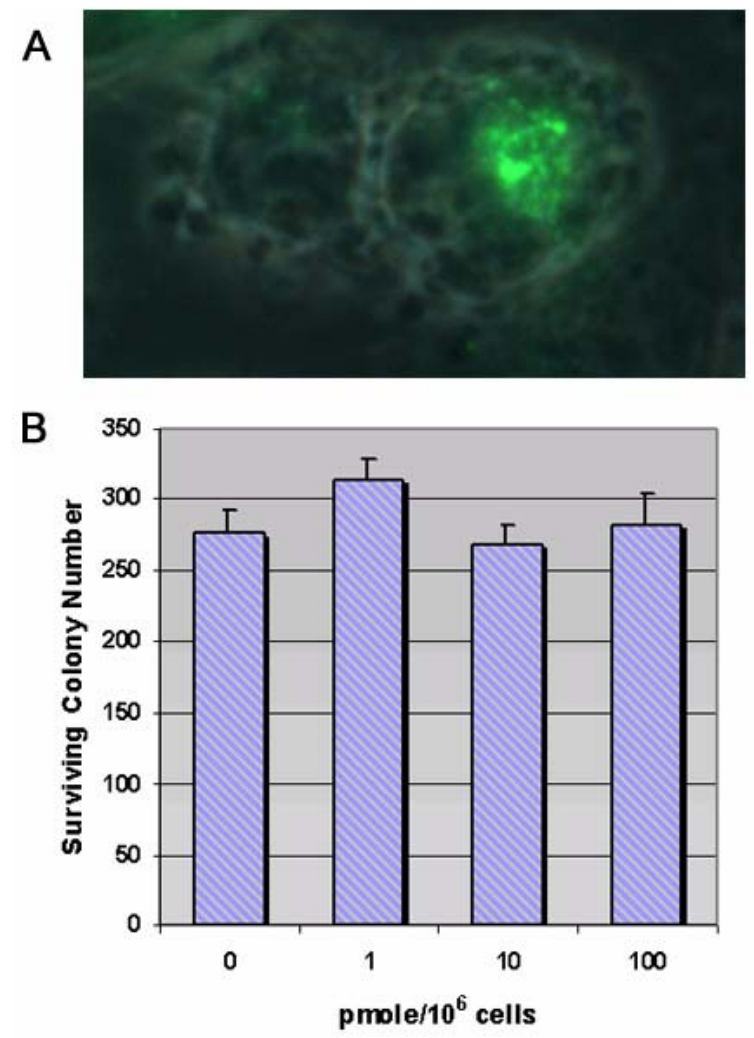

Figure 6 
Figure for Table of Contents 